\title{
Evolution of thermal properties from graphene to graphite
}

\author{
A. Alofi and G. P. Srivastava \\ School of Physics, University of Exeter, Stocker Road, Exeter EX4 4QL, United Kingdom
}

(Received 29 November 2013; accepted 29 December 2013; published online 21 January 2014)

\begin{abstract}
We report on the evolution of thermal properties from graphene to graphite as a function of layer thickness and temperature. The onset of the inter-layer compressional elastic constant $C_{33}$ and the shear elastic constant $C_{44}$ results in a large difference between the magnitudes and temperature dependencies of the specific heat and in-plane lattice thermal conductivity of bi-layer graphene (BLG) and single-layer graphene. The changes between BLG and few-layer graphene (FLG) decrease with increase in the number of layers. The cross-plane lattice thermal conductivity increases almost linearly with the number of layers in ultra-thin FLG. C 2014 AIP Publishing LLC. [http://dx.doi.org/10.1063/1.4862319]
\end{abstract}

Graphene, in addition to its remarkable electronic properties, ${ }^{1}$ is one of the materials with the highest recorded thermal conductivity values. ${ }^{2,3}$ It is also remarkable that compared to most layered systems, fabrication of singlelayer graphene (SLG), bi-layer graphene (BLG), and fewlayer graphene (FLG) can be achieved in a controlled manner. ${ }^{4}$ This strongly suggests that the FLG systems can be used to understand the fundamental mechanisms and in achieving controlled alteration of thermal conductivity along and perpendicular to the growth direction. In particular, it would be interesting to ascertain the minimum amount of thermal conductivity established when a BLG is formed.

Generally, the intrinsic ability of a material to conduct heat is altered as its dimensionality changes from twodimensional (2D) to three-dimensional (3D). Lateral (inplane) thermal conductivity in conventional semiconductors thin films tends to decrease with decreasing thickness. This is due to the domination of the boundary phonon scattering rate. ${ }^{5}$ However, an opposite dependence is observed in FLG where the thermal conductivity is reduced as the number of layers increases. ${ }^{6-8}$ As graphite is composed of multilayer graphene, it is natural to think that studying thermal properties of FLG will elucidate how the thermal conductivity and specific heat of graphene evolve into graphite-like results with increasing number of layers.

In this work, specific heat and thermal conductivity of FLG are calculated. We used the semicontinuum model proposed by Komatsu and Nagamiya, ${ }^{9}$ and employed the analytical expressions for phonon dispersion relations and vibrational density of states based on the derivations by Nihira and Iwata. ${ }^{10}$ The lattice thermal conductivity tensor was calculated within the framework of Callaway's effective relaxation time theory. ${ }^{11}$

We consider the FLG and graphite systems as an assembly of equally spaced elastic layers with compressional and shearing couplings between adjacent layers. According to the theory of elasticity, ${ }^{12}$ the strength of the inter-layer coupling in layered materials increases as the number of layers increases. Two elastic constants, $C_{33}$ and $C_{44}$, are used to describe the compressional and shearing couplings, respectively. These elastic constants are sensitive to the number of graphene layers, and any change in their values will affect the Debye-like cut-off frequencies ${ }^{10}$ and thus the phonon density of states. We adopt a convenient approach, within the semicontinuum treatment, to evaluate the effect of the $C_{33}$ and $C_{44}$ in changing the Debye-like cut-off frequencies and thus on thermal properties of FLG as the number of layers increases. Only acoustic phonon modes are considered in our calculations: in-plane longitudinal mode LA, in-plane transverse mode TA, and out-of-plane mode ZA.

The lattice specific heat at constant volume $\mathrm{C}_{\mathrm{v}}$ is calculated by using the expression

$$
\mathrm{C}_{\mathrm{v}}=k_{B} \sum_{p} \int_{\omega_{p, \min }}^{\omega_{p, \max }} \mathrm{d} \omega\left(\frac{\hbar \omega_{p}}{k_{B} T}\right)^{2} \bar{n}(\bar{n}+1) D\left(\omega_{p}\right),
$$

where $T$ is the absolute temperature, $k_{B}$ is the Boltzmann's constant, $D\left(\omega_{p}\right)$ is the density of states per mole for each polarisation $p, \bar{n}$ is the Bose-Einstein distribution function, and $\omega_{p, \min }$ and $\omega_{p, \max }$ are the lower and upper cut-offs frequencies for polarisation $p$. The density of states is given as

$$
\begin{aligned}
& p=\mathrm{LA}, \mathrm{TA}: \quad \omega \leq \omega_{z}: \quad D(\omega)=\frac{A_{m} \omega}{\pi^{2} v_{p}^{2}} \sin ^{-1}\left(\frac{\omega}{\omega_{z}}\right) ; \\
& \omega \geq \omega_{z}: \quad D(\omega)=\frac{A_{m} \omega}{2 \pi v_{p}^{2}}, \\
& p=\text { ZA and } \omega \leq \omega_{z}^{\prime}: \\
& D(\omega)=\frac{A_{m}}{2 \pi^{2} b}\left(\frac{\omega}{\omega_{z}^{\prime}}\right) \\
& \times \int_{0}^{\sin ^{-1}\left\{\left[1+\left(\zeta^{2} / 4 b^{2} \omega^{2}\right)\right]^{-1 / 2}\right\}}\left[1-\left(\frac{\omega}{\omega_{z}^{\prime}}\right)^{2}\right. \\
& \left.\times\left(1+\frac{\zeta^{2}}{4 b^{2} \omega^{2}}\right) \sin ^{2} \phi\right]^{-1 / 2} d \phi
\end{aligned}
$$

$p=\mathrm{ZA}$ and $\omega \geq \omega_{z}^{\prime}$ :

$$
\begin{aligned}
D(\omega)= & \frac{A_{m}}{2 \pi^{2} b}\left(1+\frac{\zeta^{2}}{4 b^{2} \omega^{2}}\right)^{-1 / 2} \\
& \times \int_{0}^{\pi / 2}\left[1-\left(\frac{\omega_{z}^{\prime}}{\omega}\right)^{2}\left(1+\frac{\zeta^{2}}{4 b^{2} \omega^{2}}\right)^{-1} \sin ^{2} \phi\right]^{-1 / 2} d \phi,
\end{aligned}
$$


where $A_{m}$ is the molar area, $v_{p}$ is the speed of phonons in polarisation branch $p, b$ is the bending elastic parameter which is a measure of the resistance of a graphene layer to bending, and the frequencies $\omega_{z}$ and $\omega_{z}^{\prime}$ are directly related to the shearing $\left(C_{44}\right)$ and coupling $\left(C_{33}\right)$ elastic constants as follows:

$$
\omega_{z}=2\left(\frac{C_{44}}{c^{2} \rho}\right)^{1 / 2}, \quad \omega_{z}^{\prime}=2\left(\frac{C_{33}}{c^{2} \rho}\right)^{1 / 2}
$$

where $c$ is the interlayer spacing in graphite, $\rho$ is the mass density. In terms of their physical significance, $\omega_{z}$ and $\omega_{z}^{\prime}$ are, respectively, the lower cut-off frequencies for in-plane and out-of-plane modes corresponding to the movements of rigid layers parallel and perpendicular to each other. The values of the parameters $\left(\zeta, \mu, v_{l}, v_{t}\right.$, and $\left.b\right)$ for graphite are listed in Ref. 10.

The lattice thermal conductivity tensor components can be expressed using Callaway's theory as

$$
\begin{aligned}
K_{\alpha \beta}= & \frac{\hbar^{2}}{2 A_{m} k_{B} T^{2}} \sum_{p} \int \mathrm{d} \omega \omega_{p}^{2}\left\{v_{p}(\omega)\right\}_{\alpha}\left\{v_{p}(\omega)\right\}_{\beta} \tau_{p}^{C}(\omega) \\
& \times \bar{n}(\bar{n}+1) D\left(\omega_{p}\right),
\end{aligned}
$$

where $\left\{v_{p}(\boldsymbol{q})\right\}_{\alpha}, v_{p}\{(\boldsymbol{q})\}_{\beta}$ are the components of the phonon velocity in $\alpha$ and $\beta$ directions, and $\tau_{p}^{C}(\boldsymbol{q})$ is an effective relaxation time including the momentum conserving contribution for three-phonon Normal processes. ${ }^{11,13}$ The phonon relaxation time $\tau^{-1}$ is contributed from scattering of phonons from a finite size of the sample $\tau_{\mathrm{bs}}^{-1}$, point defects $\tau_{\mathrm{pd}}^{-1}$, and anharmonicity: $\tau^{-1}=\tau_{\mathrm{bs}}^{-1}+\tau_{\mathrm{pd}}^{-1}+\tau_{\mathrm{anh}}^{-1}$. Expressions for these scattering rates are well documented and presented in our previous works. ${ }^{14,15}$

In order to deal with the in-plane and cross-plane (i.e., along the c-axis) conductivity components we need to use two sample dimensions: an in-plane length $L_{a}$ and a crossplane length $L_{c}$. Accordingly, there are two different expressions for boundary scattering

$$
\tau_{b s}(\text { in-plane })=\frac{L_{a}}{\left\{v_{p}\right\}_{a}} ; \quad \tau_{b s}(\text { cross-plane })=\frac{L_{c}}{\left\{v_{p}\right\}_{z}} .
$$

For graphite, the binding energy between adjacent layers is relatively weak compared to strong binding energy within the layers. The interaction energy of two perfectly rigid sheets are usually examined using the standard 12-6 Lennard-Jones potential between pairs of atoms with separation $c$. Usually, the parameters in that potential are employed for describing the van der Walls potential between graphene sheets per atom, which are fitted to reproduce the interlayer distance and the elastic constant $C_{33}$ for graphite. ${ }^{16,17}$ The value of $C_{44}$ could be attained experimentally. We will employ a simple alternative scheme to obtain values of $C_{33}$ and $C_{44}$ as a function of the number of layers in FLG.

The shear-mode frequency $\omega_{z}$ for FLG sheets as a function of the number of layers were measured by Tan et al. ${ }^{18}$ using Raman spectroscopy. The lower cut-off out-of-plane frequency $\omega_{z}^{\prime}$ for SLG, BLG, and tri-layer graphene (TLG) sheets are obtained from Ref. 19. Using suitable fit of these data, we determine $C_{33}$ and $C_{44}$ for FLG of different number

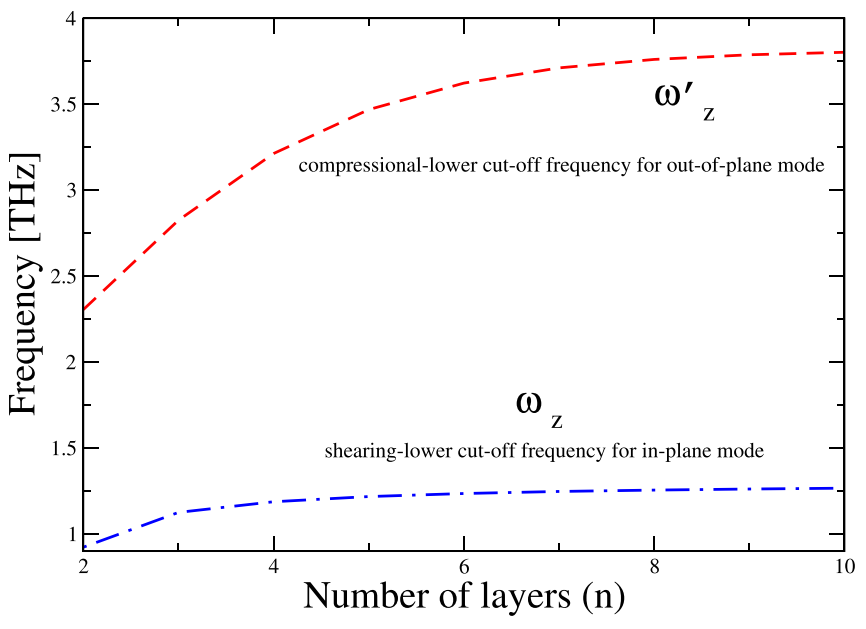

FIG. 1. Variation of lower cut-off frequencies $\omega_{z}$ and $\omega_{z}^{\prime}$ with the number of graphene atomic layers.

of layers with the help of Eq. (5). From Fig. 1, it can be noticed that both $\omega_{z}$ and $\omega_{z}^{\prime}$ have fully saturated to the graphite values presented in Ref. 10 for $n \simeq 10$. However, $\omega_{z}$ saturates more rapidly than $\omega_{z}^{\prime}$. As discussed in Ref. 15, phonon conductivity calculations were made by considering the point-defect parameter $A_{d}=4.5 \times 10^{-5}$ and the anharmonic scattering parameters: $B_{U}=3.18 \times 10^{-25} \mathrm{sK}^{-3}$, and $B_{N}=2.12 \times 10^{-25} \mathrm{sK}^{-3}$.

Figure 2 shows the variation of specific heat $C_{v}$ with temperature for multilayer graphene sheets and bulk graphite. For SLG, there are no shearing and compressional couplings between layers, which means that $C_{33} \approx 0$ and $C_{44} \approx 0$, and hence $\omega_{z} \rightarrow 0$ and $\omega_{z}^{\prime} \rightarrow 0$. At low temperatures $(<60 \mathrm{~K})$, a large difference can be seen between the specific heat of SLG and BLG sheets. It can be interpreted to arise from the presence of layer couplings, i.e., due to non-zero values of $C_{33}$ and $C_{44}$, in BLG. There is a remarkable change in the temperature dependence of $\mathrm{C}_{\mathrm{v}}$ from $T^{1.1}$ for SLG to $T^{2.8}$ for BLG.

The in-plane phonon conductivity calculations were made by considering a sample of size $L_{a}=2.9 \mu \mathrm{m}$. Figure 3(a) shows the calculated thermal conductivity for SLG, BLG, and TLG along the graphite basal planes. These calculations reveal that the in-plane thermal conductivity

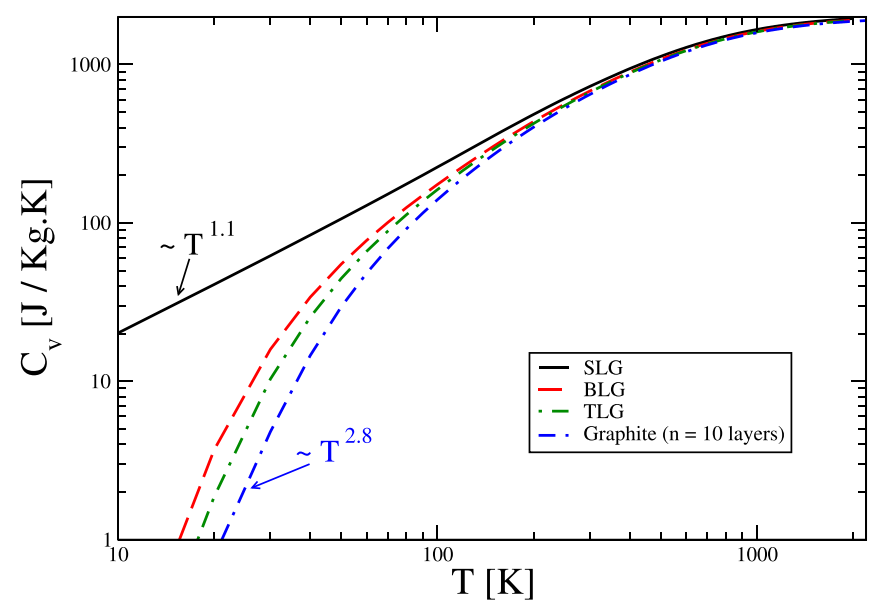

FIG. 2. Specific heat $\mathrm{C}_{\mathrm{v}}$ for multilayer graphene sheets. 

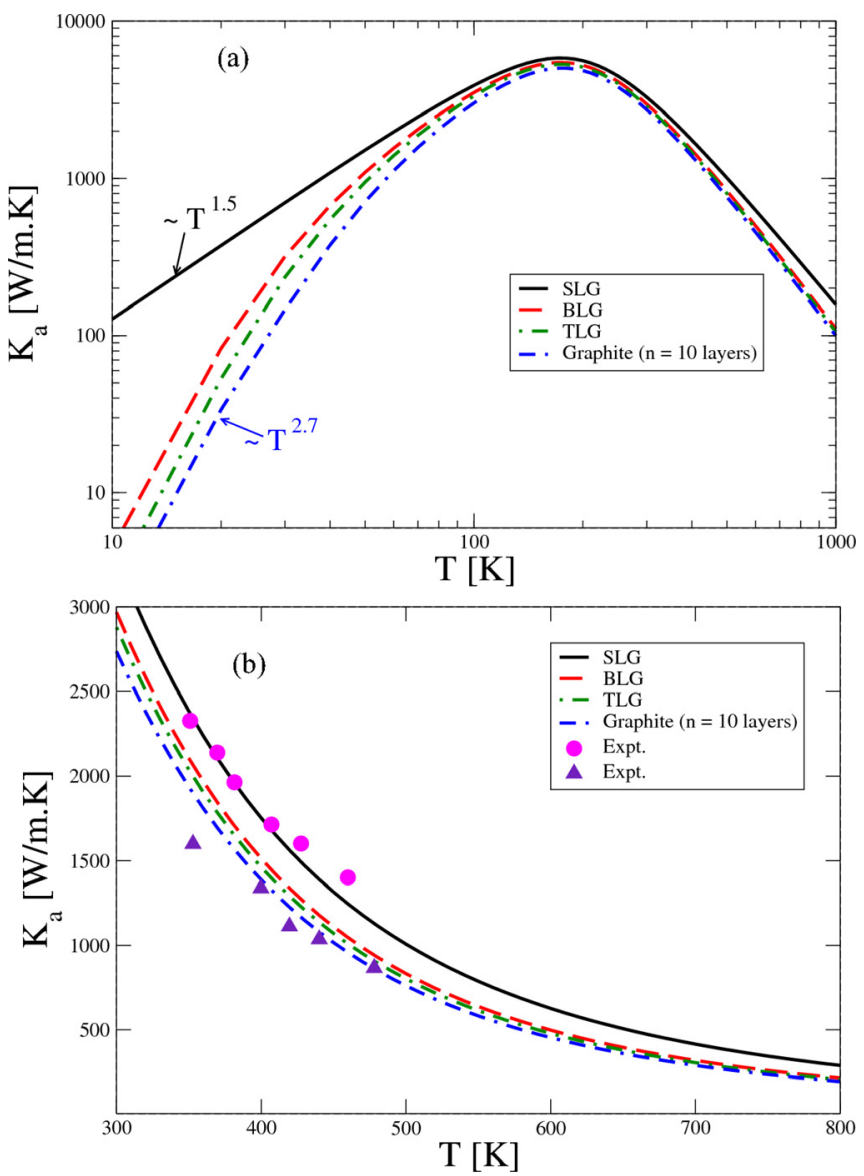

FIG. 3. (a) In-plane thermal conductivity for multilayer graphene sheets. (b) Comparison of computed results with experimental results for SLG and graphite. The symbols represent the experimental measurements: SLG (circles) (Ref. 21); and graphite basal planes (up triangles) (Ref. 22).

$\left(K_{a} \equiv K_{x x}=K_{y y}\right.$ ) decreases monotonically as the number of layers increases. There is a change of low-temperature dependence from $T^{1.5}$ to $T^{2.7}$ as the dimensionality evolves from strictly two-dimensional for SLG to three-dimensional for bulk graphite. The $T^{2.7}$ temperature dependence of the basal plane thermal conductivity of graphite agrees with experimental measurement in Ref. 20. Figure 3(b) shows the thermal conductivities above room temperature along with experimental data available for SLG and bulk graphite. We notice that the difference between the thermal conductivities of SLG and FLG diminishes with increasing temperature, consistent with the trend noted in another theoretical work. ${ }^{7}$

It is more interesting to examine the variation of the cross-plane conductivity $\left(K_{c} \equiv K_{z z}\right)$ as a function of the number of layers $n$. Fig. 4 shows an increase of $K_{c}$ as the number of layers increases. The boundary length along c-axis, $L_{c}$, for FLG was taken as $L_{c}=(n-1) c$. Of course, $K_{c}=0$ for SLG $(n=1)$. For BLG, the $K_{c}$ starts to emerge with very low values and weak temperature dependency. Higher values of the conductivity are established for FLG. However, for a stand-alone $n$-layer FLG with the boundary length set to $L_{c}=(n-1) c$, the temperature dependency remains very weak below room temperature, although there appears to be a mildly increased temperature dependence as $n$ increases. This can be clearly seen from the results for FLG with $n=3$, 4, and 10. For BLG as well as FLG, there is a clear temperature dependence and bunching of the conductivity above room temperature, due

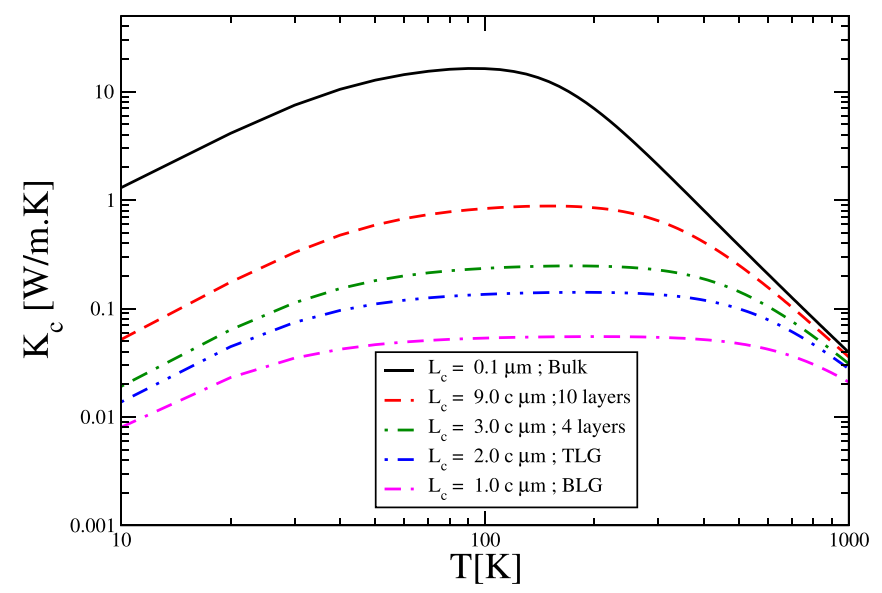

FIG. 4. Thermal conductivity along c-axis $K_{c}$ for multilayer graphene sheets.

to increasing role of anharmonic phonon interactions. A finite-size graphite sample can be considered as several FLG stacked upon each other. Calculations for graphite with $L_{c}=0.1 \mu \mathrm{m}$ suggest that there is a well-established maximum in the $K_{c} v s$. $T$ curve at around $100 \mathrm{~K}$. The conductivity of bulk graphite $K_{c}$ at $100 \mathrm{~K}$ and for $L_{c}=0.1 \mu \mathrm{m}$ is three orders of magnitude higher than that for BLG, and more than an order of magnitude larger than that for FLG with $n=10$ and $L_{c}=9 c$. This vindicates the well-known important role of sample size, via boundary scattering of phonons, in determining the magnitude of low-temperature conductivity.
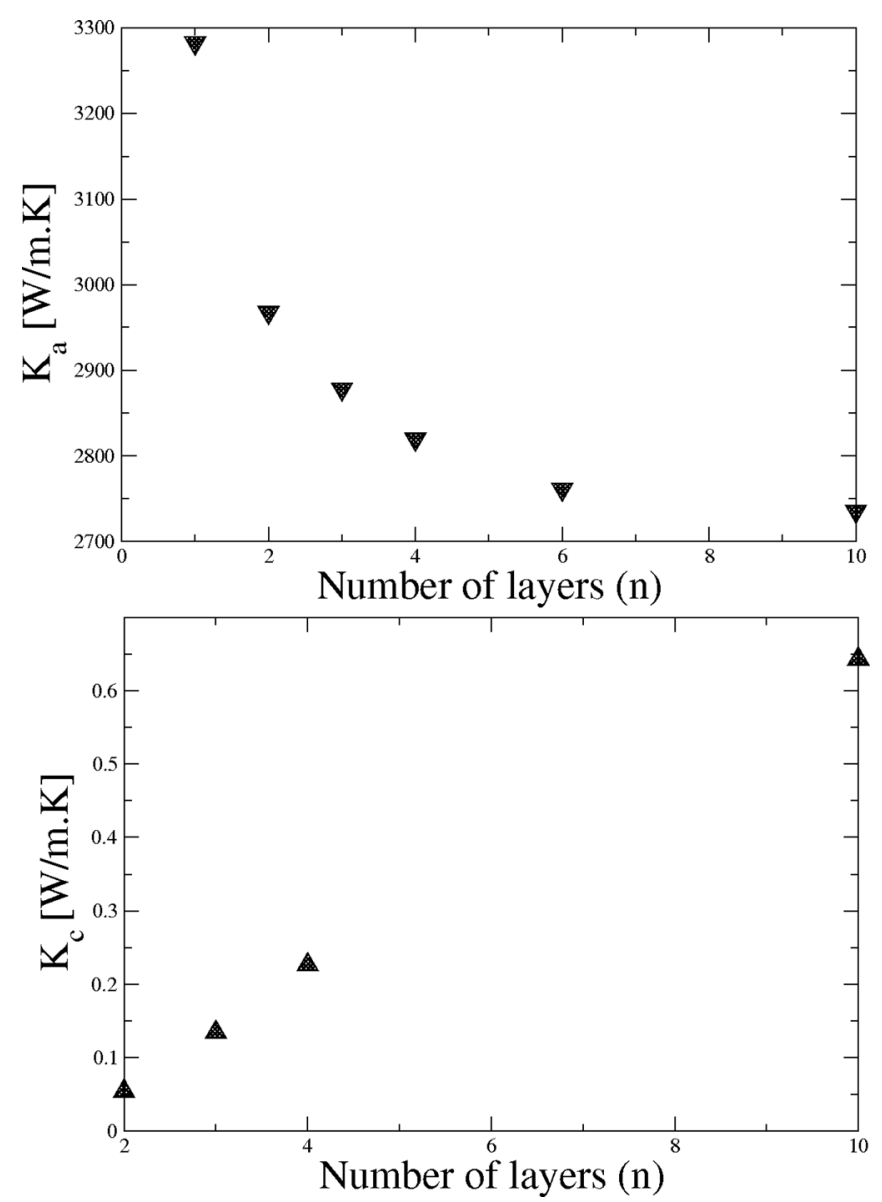

FIG. 5. Variation of the room-temperature results for $K_{a}$ (upper panel) and $K_{c}$ (lower panel) as a function of the number of graphene layers. 
The changes in the room-temperature values of $K_{a}$ and $K_{c}$ as a function of the number of atomic planes $n$ in FLG are shown in Fig. 5. Compared to SLG, $K_{a}$ of BLG is reduced by more than $300 \mathrm{~W} \mathrm{~m}^{-1} \mathrm{~K}^{-1}$. The conductivity $K_{a}$ progressively decreases as the number of layers $n$ increases beyond 2 , nearly saturating at the graphite value for $n=10$.

The changes predicted by our theory for $n=2,3$, and 4 are consistent with the measurements made by Ghosh et al. ${ }^{6}$ However, a direct comparison of our results with those in Ref. 6 is not possible for two reasons: there is a large error margin in the experimental measurements (e.g., $3000-5000 \mathrm{~W} \mathrm{~m}^{-1} \mathrm{~K}^{-1}$ at room temperature for SLG), and the concentration of defects in the samples of different layer numbers is unknown.

In contrast, the variation in $K_{c}$ is almost linear for ultrathin FLG (at least up to the layer index $n=4$ ). In other words, $K_{c}$ is governed by $L_{c}$. The difference between $K_{c}$ for FLG and bulk graphite is mainly due to their sample thicknesses: for stand-alone FLG with $n=10$, the crossdirectional sample length is $L_{c}=9 c$, and for bulk graphite, we have considered a film of thickness $0.1 \mu \mathrm{m}$.

The establishment of the finite and temperature independent cross-plane conductivity magnitude $0.05 \mathrm{~W} \mathrm{~m}^{-1} \mathrm{~K}^{-1}$ for BLG is a very interesting result, and points towards a fundamental aspect of the thermal physics of layered materials in general. Based upon our result, we suggest that a finite and temperature independent amount of cross-plane lowtemperature lattice thermal conductivity should be observed for all materials that can be fabricated as stand-alone bilayered systems.

With the development of appropriate experimental techniques, such as the time-domain thermoreflectance (TDTR) method $^{23}$ for measuring heat conduction across metal/ graphene/oxide interfaces, we anticipate that our theoretical predictions of the cross-plane conductivity results for FLG can be tested in near future.
Ayman Salman Alofi gratefully acknowledges financial support from Taibah University, Madinah, Saudi Arabia.

${ }^{1}$ K. S. Novoselov, A. K. Geim, S. V. Morozov, D. Jiang, Y. Zhang, S. V. Dubonos, I. V. Grigorieva, and A. A. Firsov, Science 306, 666 (2004).

${ }^{2}$ D. L. Nika, E. P. Pokatilov, A. S. Askerov, and A. A. Balandin, Phys. Rev. B 79, 155413 (2009).

${ }^{3}$ S. Ghosh, I. Calizo, D. Teweldebrhan, E. P. Pokatilov, D. L. Nika, A. A. Balandin, W. Bao, F. Miao, and C. N. Lau, Appl. Phys. Lett. 92, 151911 (2008).

${ }^{4}$ W. Bao, F. Miao, Z. Chen, H. Zhang, W. Jang, C. Dames, and C. N. Lau, Nat. Nanotechnol. 4, 562 (2009).

${ }^{5}$ W. Liu and M. Asheghi, J. Heat Transfer 128, 75 (2005).

${ }^{6}$ S. Ghosh, W. Bao, D. L. Nika, S. Subrina, E. P. Pokatilov, C. N. Lau, and A. A. Balandin, Nat. Mater. 9, 555 (2010).

${ }^{7}$ D. Singh, J. Y. Murthy, and T. S. Fisher, J. Appl. Phys. 110, 044317 (2011).

${ }^{8}$ Z. Wei, Z. Ni, K. Bi, M. Chen, and Y. Chen, Carbon 49, 2653 (2011).

${ }^{9}$ K. Komatsu and T. Nagamiya, J. Phys. Soc. Jpn. 6, 438 (1951).

${ }^{10} \mathrm{~T}$. Nihira and T. Iwata, Phys. Rev. B 68, 134305 (2003).

${ }^{11}$ J. Callaway, Phys. Rev. 113, 1046 (1959).

${ }^{12}$ L. D. Landau and E. M. Lifshitz, Course of Theoretical Physics, Vol. 7: Theory of Elasticity (Pergamon, New York, 1986; Nauka, Moscow, 1987).

${ }^{13}$ G. P. Srivastava, The Physics of Phonons (Adam and Hilger, Bristol, 1990).

${ }^{14}$ A. Alofi and G. P. Srivastava, J. Appl. Phys. 112, 013517 (2012).

${ }^{15}$ A. Alofi and G. P. Srivastava, Phys. Rev. B 87, 115421 (2013).

${ }^{16}$ R. Saito, R. Matsuo, T. Kimura, G. Dresselhaus, and M. S. Dresselhaus, Chem. Phys. Lett. 348, 187 (2001).

${ }^{17}$ Y. Shibuta and J. A. Elliott, Chem. Phys. Lett. 512, 146 (2011).

${ }^{18}$ P. H. Tan, W. P. Han, W. J. Zhao, Z. H. Wu, K. Chang, H. Wang, Y. F. Wang, N. Bonini, N. Marzari, N. Pugno, G. Savini, A. Lombardo, and A. C. Ferrari, Nat. Mater. 11, 294 (2012).

${ }^{19}$ E. Faizabadi and F. Karbalaii, Proc. SPIE 8101, 81010S (2011).

${ }^{20}$ M. G. Holland, C. A. Klein, and W. D. Straub, J. Phys. Chem. Solids 27, 903 (1966).

${ }^{21}$ S. Chen, A. L. Moore, W. Cai, J. W. Suk, J. An, C. Mishra, C. Amos., C. W. Magnuson, J. Kang, L. Shi, and R. S. Ruff, ACS Nano 5, 321 (2011).

${ }^{22}$ G. A. Slack, Phys. Rev. 127, 694 (1962).

${ }^{23}$ Y. K. Koh, M.-H. Bae, D. G. Cahill, and E. Pop, Nano Lett. 10, 4363 (2010). 